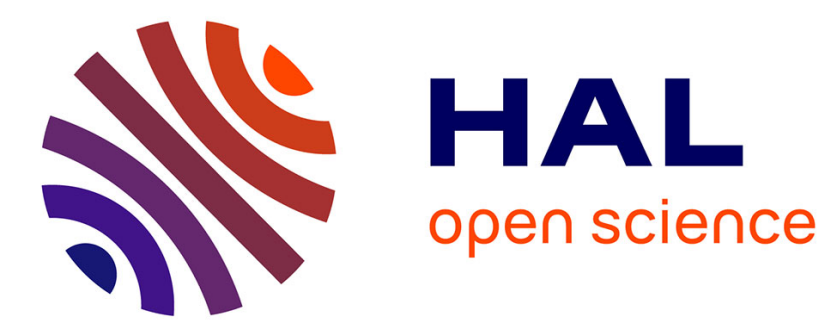

\title{
Interplay between Gyrokinetic Turbulence, Flows, and Collisions: Perspectives on Transport and Poloidal Rotation
}

Guilhem Dif-Pradalier, Virginie Grandgirard, Yanick Sarazin, Xavier Garbet, Philippe Ghendrih

\section{To cite this version:}

Guilhem Dif-Pradalier, Virginie Grandgirard, Yanick Sarazin, Xavier Garbet, Philippe Ghendrih. Interplay between Gyrokinetic Turbulence, Flows, and Collisions: Perspectives on Transport and Poloidal Rotation. Physical Review Letters, 2009, 103, pp.065002. 10.1103/PhysRevLett.103.065002 . cea-01468365

\section{HAL Id: cea-01468365 https://hal-cea.archives-ouvertes.fr/cea-01468365}

Submitted on 15 Feb 2017

HAL is a multi-disciplinary open access archive for the deposit and dissemination of scientific research documents, whether they are published or not. The documents may come from teaching and research institutions in France or abroad, or from public or private research centers.
L'archive ouverte pluridisciplinaire $\mathbf{H A L}$, est destinée au dépôt et à la diffusion de documents scientifiques de niveau recherche, publiés ou non, émanant des établissements d'enseignement et de recherche français ou étrangers, des laboratoires publics ou privés. 


\title{
Interplay between Gyrokinetic Turbulence, Flows, and Collisions: Perspectives on Transport and Poloidal Rotation
}

\author{
G. Dif-Pradalier, ${ }^{1,2}$ V. Grandgirard, ${ }^{1}$ Y. Sarazin, ${ }^{1}$ X. Garbet,${ }^{1}$ and Ph. Ghendrih ${ }^{1}$ \\ ${ }^{1}$ Association Euratom-CEA, CEA/IRFM, F-13108 St. Paul-lez-Durance cedex, France \\ ${ }^{2}$ Center for Astrophysics and Space Sciences, UCSD, La Jolla, California 92093, USA \\ (Received 15 January 2009; revised manuscript received 15 April 2009; published 4 August 2009; \\ publisher error corrected 4 September 2009)
}

\begin{abstract}
The impact of ion-ion collisions on confinement is investigated with the full- $f$ and global gyrokinetic GYSELA code through a series of nonlinear turbulence simulations for tokamak parameters. A twofold scan in the turbulence drive and in collisionality is performed, highlighting (i) a heat transport expressed in terms of critical quantities - threshold and exponent, (ii) a first evidence of turbulent generation of poloidal momentum, and (iii) the dominance of mean flow shear, mediated through the turbulent corrugation of the mean profiles, with regard to the oft-invoked zonal flow shear.
\end{abstract}

PACS numbers: 52.65.Tt, 52.30.Gz, 52.35.Mw, 52.35.Ra

Gyrokinetic theory [1] provides today's deepest insight into turbulence-related problems in plasma physics, especially allowing for a very accurate description of turbulent transport. Neoclassical theory [2] was earlier coined to describe the effects of binary Coulomb collisions in an inhomogeneous toroidal magnetic field. Elucidating the interplay between turbulence and collisions is a subject of great current focus for modeling since recent evidence from both experiments [3,4] and simulations [5,6] has started to emphasize its special relevance for the onset and control of enhanced confinement regimes. A paradigm is that a highly sheared $\mathbf{E} \times \mathbf{B}$ flow can lead to better confinement through decorrelation of turbulent fluctuations or decrease in radial correlation lengths. Accordingly, the fundamental importance of the radial electric field [3] or of zonal flows [7] is widely emphasized.

Zonal flows (ZFs) are low frequency turbulence-induced axisymmetric sheared $\mathbf{E} \times \mathbf{B}$ flows. Along with the mean flows (MFs) - temporally averaged $\mathbf{E} \times \mathbf{B}$ flows over a collision time - they dynamically participate into turbulence self-regulation and are linearly damped through collisions alone. In a standard predator-prey picture, the ZFs (predator) regulate the turbulence (prey); the turbulent transport itself may thus depend on neoclassical effects. Accordingly, collisional enhancement of turbulent transport has recently been reported in fluid [6] and quasilocal $(\delta f)$ gyrokinetic simulations [5] of ion temperature gradient (ITG) turbulence including ion-ion collisions.

Collisional processes are also central for an accurate calculation of the radial electric field $E_{r}$, mainly through the determination of the poloidal rotation $v_{\theta}$. The accepted idea [3] is that the experimentally reported deviations of $v_{\theta}$ from its neoclassical expectation at the low to high confinement transition is a precursor for the observed large evolution of $E_{r}$. Understanding the physical mechanisms which allow for the generation of poloidal momentum could prove especially crucial to understand the formation and the dynamics of internal transport barriers (ITBs) and to shed a quantitative insight on the physics of the $L-H$ transition. An accurate description of the radial force balance $E_{r}-v_{\varphi} B_{\theta}+v_{\theta} B_{\varphi}=\nabla p / n Z e$ is thus of prime importance for an emerging generation of gyrokinetic codes which calculate the full distribution function (see [8] and references therein). In such codes alone is $E_{r}$ indeed selfconsistently evolved [9] and coupled to the mean profile dynamics, through either the evolution of the mean pressure gradient $\nabla p$ (thermodynamic force) or that of the flows (poloidal and toroidal $v_{\varphi}$ ).

In this Letter, (i) we revisit the question of the collisional enhancement of turbulent transport near criticality, and (ii) we report the first quantitative prediction for the poloidal rotation in turbulent regimes including neoclassical effects using the GYSELA code [10]. These results are based on a series of nonlinear gyrokinetic simulations including ion-ion collisions in global realistic magnetic geometry and representative core plasma parameters. These simulations have been performed in both neoclassical and turbulent regimes, and a convergence study has been performed, at different values of the small tokamak parameter $\rho_{\star}=$ $\rho_{i} / a, a$ being the minor radius and $\rho_{i}$ the ion gyroradius. For neoclassical simulations, the temperature gradient $R_{0} / L_{T}=3$ is chosen such that the system remains below the linear instability threshold. This parameter represents the strength of the turbulence drive. The numerical values reported below are given at midradius $r=0.5 a: R_{0} / L_{n}=$ 2.2, $T_{i} / T_{e}=1, \quad \epsilon=r / R=0.17, \quad q=1.4, \quad$ and $s=$ $(r / q) d q / d r=0.78$. The neoclassical problem is axisymmetric and the grid size at $\rho_{\star}=1 / 256$ involves over $10^{9}$ grid points on a half-torus $\left(r, \theta, \varphi, v_{\|}, \mu\right)=$ $(256,256,8,128,16)$ mesh. Here $q$ is the safety factor, $R=$ $R_{0}+r \cos \theta$ the major radius, $\epsilon$ the inverse aspect ratio, $T_{i}$ and $T_{e}$ the ion and electron temperatures, respectively, and $\mu$ the adiabatic invariant. Collisionality reads: $\nu_{\star}=$ $\epsilon^{-3 / 2} q R_{0} \nu_{i i} / v_{T}, v_{T}=\sqrt{T / m}$ being the thermal velocity and $\nu_{i i}$ the ion-ion collision frequency. 


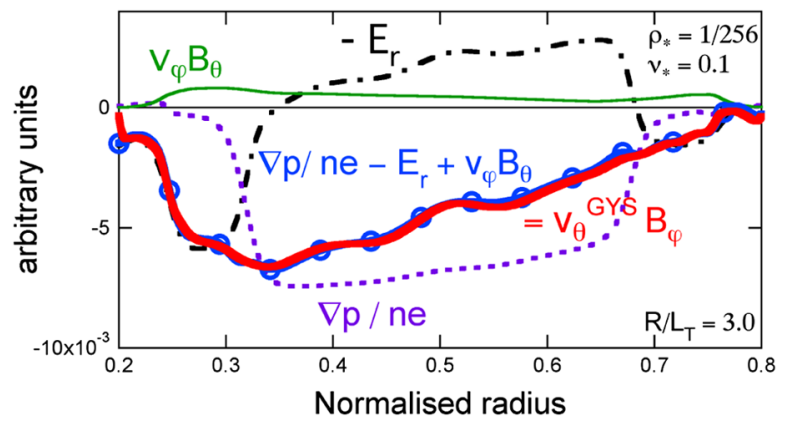

FIG. 1 (color online). Accurate satisfaction of the equilibrium radial force balance, at any given simulation time.

In particular (see Refs. $[11,12])$, the dependencies on $\nu_{\star}$ and $\epsilon$ of the neoclassical heat transport $[13,14]$ and poloidal rotation [15] have been accurately verified in the low and intermediate tokamak-relevant banana and plateau regimes along with the collisional damping [16] of axisymmetric mean and zonal flows-which survive the collisionless linear Landau damping process. As the last mandatory check, $E_{r}$ self-consistently adjusts within a few percent according to the radial force balance in either neoclassical or turbulent regimes, for all collisionalities. An example in the neoclassical regime is displayed in Fig. 1. The poloidal velocity is shown, calculated by two means: (i) self-consistently within GYSELA $v_{\theta}^{\text {GYS }}$ from the distribution function as the sum of the poloidal $\mathbf{E} \times \mathbf{B}$, curvature, grad- $B$, and magnetization flows and (ii) by means of the force balance $\nabla p / n e-E_{r}+v_{\varphi} B_{\theta}$, each of the latter terms being independently plotted. This ability of full- $f$ codes to accurately compute the long wavelengths of $\phi$ (the mean $E_{r}$ ) has recently been questioned [17]; we show excellent agreement with the radial force balance when using conventional first order gyrokinetic equations.

Simulations of tokamak turbulence are now performed for the same representative core plasma parameters. Scanning the strength of the turbulence drive and the collisionality allows one to assess the possible interplay between collisions, flows, and turbulence for transport and poloidal rotation. Four different $R / L_{T}$ values are investigated, either inside the so-called "Dimits upshift" (below the ITG nonlinear collisionless threshold [18] $)\left(R / L_{T}=5.6\right)$, close above threshold (6.3), and further above (7.6 and 10.9). Each value is a different simulation; for each simulation, $\nu_{\star}$ is stepped down twice in the banana regime after convergence is reached: $\nu_{\star}=0.5,0.1,0.05$. One more simulation $\left(\nu_{\star}=0.01, R / L_{T}=7.6\right)$ is performed. Each of these are run at $\rho_{\star}=1 / 128$ on a half-torus $\left(r, \theta, \varphi, v_{\|}, \mu\right)=$ $(256,256,64,64,8)$ grid. Two additional simulations close above this threshold are also run on a half-torus highresolution grid $\left(r, \theta, \varphi, v_{\|}, \mu\right)=(512,512,128,128,16)$ at $\rho_{\star}=1 / 256$. In each, the two values of the collisionality $\nu_{\star}=0.5$ and 0.05 are scanned.

The results are summarized in Fig. 2 [respectively, Fig. 4], where small markers represent the $\rho_{\star}=1 / 128$ simulations and large markers the $\rho_{\star}=1 / 256$ high-

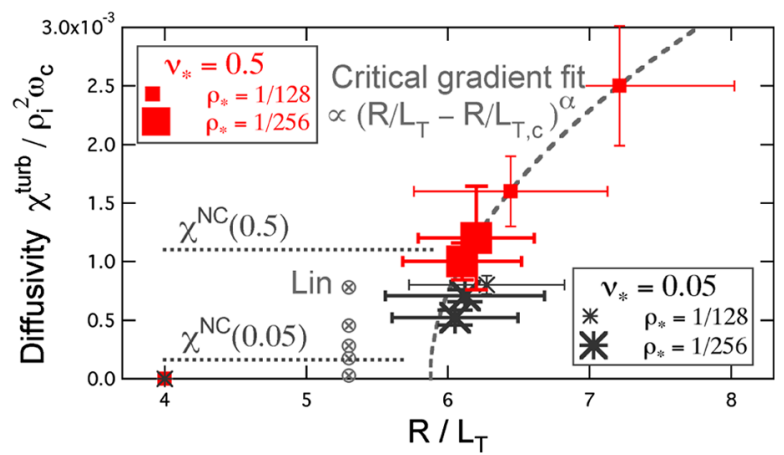

FIG. 2 (color online). A cartography in $\left(R / L_{T}, \nu_{\star}\right)$ space of the turbulent heat flux $\chi^{\text {turb }}$. The result in Ref. [5] is shown, as is the neoclassical heat flux $\chi^{\mathrm{NC}}$ for the two reported collisionalities.

resolution ones. For clarity, we display in Fig. 2 only the $\nu_{\star}=0.5$ and 0.05 cases. Each symbol corresponds to a radial and temporal average of both the turbulent heat diffusivity and the drive of the turbulence [respectively, poloidal velocity and shear $\left.\gamma\left(v_{\theta}\right)=r \partial_{r}\left(v_{\theta} / r\right)\right]$. Temporal averages are performed in the statistical steady-turbulent regime over one to three collision times $\Delta t$. Radial averages are performed far from the boundary layers, between 0.4 and 0.7 in normalized radius: $\Psi \in \mathbb{1}_{r}=[0.4,0.7]$. While investigating the different sources of "uncertainty" in these actual averages, both the temporal fluctuations $\delta \chi^{t}$ of the turbulent heat diffusivity [respectively, $\delta v_{\theta}^{t}$ ] during $\Delta t$ and the variance of the fluctuations $\delta\left\langle R / L_{T}\right\rangle$ in the drive of the turbulence must be considered. Indeed, at each radial location, as fronts propagate radially, the local drive seen by the turbulence fluctuates by $\delta\left\langle R / L_{T}\right\rangle$. These fluctuations follow from the intrinsically global character of the simulation and the existence of avalanchelike large-scale events associated to large heat outflows radially propagating on large distances (several correlation lengths) at fractions of the diamagnetic velocity $v_{\star}=\rho_{\star} v_{T}$ [19]. They lead to the horizontal bars in Fig. 2, the vertical bars capturing the temporal fluctuations $\delta \chi^{t}$ [respectively, $\delta v_{\theta}^{t}$ ].

Note that we purposely make use of a quasilocal diffusion coefficient $\chi$, further complexity of the system being imparted to these fluctuation bars. This approach allows for an up-front comparison with existing quasilocal transport models. The additional complexity displayed in a statistical flux-driven system, nondiffusive (non-Gaussian or Markovian) statistics [20], nonlocal dynamics [21,22] (avalanching and spreading), advocates for a change in the modeling of the heat flux: $Q=-\int \aleph\left(x, x^{\prime}\right) \nabla T\left(x^{\prime}\right) d x^{\prime}$ and will give rise to forthcoming work.

In the spirit of phase transitions, we define (i) a critical gradient $R / L_{T, c}$ for each collisionality as the value for which the turbulent transport overcomes neoclassical transport (dotted lines in Fig. 2) and (ii) a critical exponent $\alpha$ quantifying the resilience (or stiffness) of the transition: $\chi^{\text {turb }} \propto\left(R / L_{T}-R / L_{T, c}\right)^{\alpha}$. In the least-squares sense, $\alpha=$ $0.5\left({ }_{-0.25}^{+0.11}\right)$ and $R / L_{T, c}=5.9\left({ }_{-0.17}^{+0.01}\right)$. This latter value is consistent with the usual nonlinear threshold at vanishing 
collisionality [18], the uncertainties on the two critical quantities reflecting the existence of the temporal fluctuations $\delta \chi^{t}$. An equivalent [21] and certainly deeper standpoint consists in expressing $\alpha$ in terms of the increment in power $\delta P$ necessary to tweak the profiles: $\left(R / L_{T}-\right.$ $\left.R / L_{T, c}\right) \propto \delta \mathcal{P}^{\beta}$. Here $\beta=(1+\alpha)^{-1} \approx \frac{2}{3}$ sits close to the crossover point between weak and strong turbulence (the usual weak turbulence scaling would predict $\beta \leq \frac{1}{2}$ and thus a stronger stiffness at marginality yet less enduring as one moves farther from $R / L_{T, c}$ ). We believe that the experimental testing of this prediction could shed an interesting insight on the physics near criticality.

Below and close to criticality, turbulent transport is expected to be most sensitive to $\mathbf{E} \times \mathbf{B}$ flow regulation, via shear decorrelation of turbulent eddies. From the mean values of $\chi^{\text {turb }}$ in Fig. 2, one observes an enhancement of turbulent transport with $\nu_{\star}$, in qualitative agreement with earlier quasilocal results [5] ("Lin" in Fig. 2). The main novelty here comes from the ability to describe the mean profile dynamics [23] (inclusion of the MFs and selfconsistent dynamics of $E_{r}$ ) which stems from the conjunction of a full- $f$ capability and an incoming heat flux from thermal baths at the radial edges. Such a system is intrinsically statistical, prominently highlighting self-organized near-critical transport-long tail in the autocorrelation function, $1 / f$ spectra, and Hurst exponents of order unity [12] — featuring the crucial role of temperature profile stiffness near marginality, as reported experimentally. The system self-organizes sitting close to criticality, so that large discrepancies in transport can occur with a modest change in the turbulence drive. Subcritical regions $\left(<R / L_{T, c}\right)$ radially coexist with critical and supercritical regions so that a local analysis at a local $R / L_{T}$ will fail to capture this complexity.

We find that (i) the shear associated with the MF is at least comparable to that of the ZFs (see Fig. 3 and discussion below). Also, (ii) the transport decrease $Q=$ $\left\langle\delta p^{2}\right\rangle^{1 / 2}\left\langle\delta v_{E, r}^{2}\right\rangle^{1 / 2} \sin \delta_{p_{k}, \phi_{k}}$ when stepping down the collisionality equally occurs through the decrease in the amplitudes of the pressure and radial $\mathbf{E} \times \mathbf{B}$ velocity $\left\langle\delta p^{2}\right\rangle^{1 / 2}$ and $\left\langle\delta v_{E, r}^{2}\right\rangle^{1 / 2}$ and the dephasing of these: $\delta_{p_{k}, \phi_{k}}$. Though some differences may exist between the calculated values and the weak turbulence prediction for the critical exponents $\alpha$ or $\beta$, (iii) the weak turbulence scaling $\chi^{\text {turb }} \propto \delta \phi^{2}$, regardless of transient departures from it when stepping down the collisionality, is robustly recovered in the long run (beyond a collision time). On the other hand, (iv) our results suggest a departure from the conventional predatorprey prediction [24]: $\delta \phi^{2} \propto \nu_{i i}$. A possible reconciliation with this model may consist of its further development to self-consistently include the distance to criticality when calculating the growth of seed fluctuations. At last, as one may ask to go beyond the now usual ZF-dominated paradigms, full- $f$ flux-driven numerical experiments could prove especially useful to investigate new pieces of physics: e.g., MF effects (see the following) or the very funda-

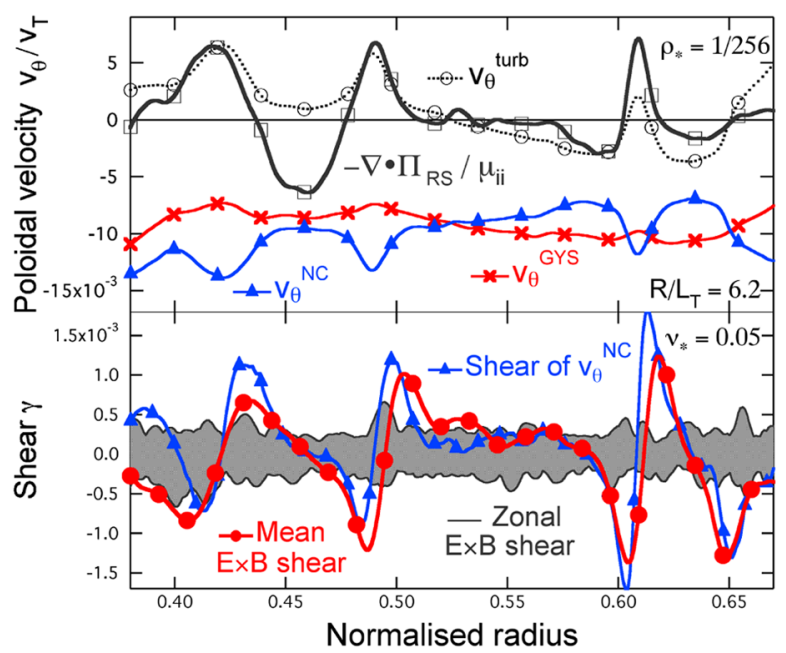

FIG. 3 (color online). Flux-surface and time-averaged $(\Delta t=$ $\nu_{i i}^{-1}$ ) computation at low collisionality in the source-free region of the poloidal velocity (top), its turbulent and neoclassical parts along with different shears (bottom), neoclassical and $\mathbf{E} \times \mathbf{B}$.

mental yet unaddressed questions of the influence of collisions and flows on avalanche statistics or the link between flow structure and dissipation profiles. These questions will be reported elsewhere.

As a mirror problem to the collisional enhancement of turbulent transport, we now address the question of the turbulent generation of poloidal rotation. It is widely believed that the collisional interaction between trapped and passing particles in the banana regime and magnetic pumping in all collisionality regimes heavily damp the poloidal flow towards its neoclassical value. Yet, some recent theories [25] have pointed out the possible generation of poloidal momentum from turbulent Reynolds stresses in a bifurcation scenario in which poloidal flows could act as a precursor in the transition from low to high confinement. Such calculations require the self-consistent evaluation of these stresses and their contribution to large-scale poloidal flows; as such they may only be consistently addressed by full- $f$ computations.

From the momentum equation, the long-time equilibrium of the flux-surface averaged poloidal velocity reads $\left\langle v_{\theta}\right\rangle=\left\langle v_{\theta}^{\mathrm{NC}}\right\rangle-\partial_{r}\left[r^{2}\left\langle\tilde{v}_{r} \tilde{v}_{\theta}\right\rangle\right] /\left(r^{2} \mu_{i i}\right)$. Out of the scan in $\left(R / L_{T}, \nu_{\star}\right)$ space, we highlight in Fig. 3 the results from one of the high-resolution $\rho_{\star}=1 / 256$ simulations, in the banana regime $\nu_{\star}=0.05$ and close to marginality $R / L_{T}=$ 6.2. Each term in the latter equation is displayed: the lefthand side term-again labeled $v_{\theta}^{\mathrm{GYS}}$ —is the self-consistent computation in the different turbulent regimes, including collisions. The second term corresponds to the neoclassical prediction and is labeled $v_{\theta}^{\mathrm{NC}}=K_{1} \nabla T / e B$. We evaluate this quantity using the following procedure: (i) the $K_{1}$ coefficient-with its radial dependence-is evaluated through dedicated simulations in the neoclassical regime [12]. Then, (ii) from the turbulent simulations from which we have evaluated $v_{\theta}^{\text {GYS }}$, we compute the actual $\nabla T$ value - these two sets of simulations having the exact same 


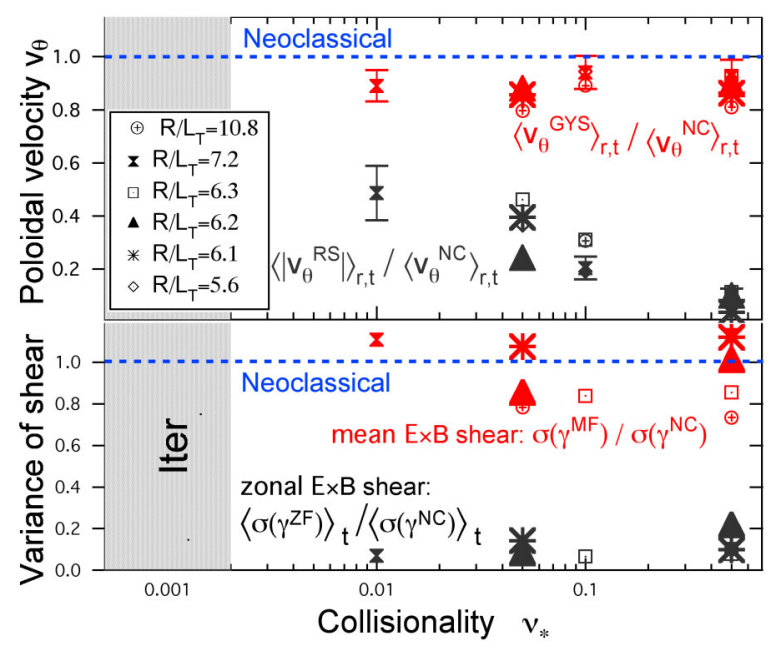

FIG. 4 (color online). Time and radial average $\left(\rrbracket_{r}=[0.4,0.7]\right)$ of $v_{\theta}$ (top) and the variance of the $\mathbf{E} \times \mathbf{B}$ shears (bottom) in the source-free region-a cartography in $\left(R / L_{T}, \nu_{\star}\right)$ space.

parameters apart from the actual value of $R / L_{T}$. We then (iii) compute $v_{\theta}^{\mathrm{NC}}=K_{1} \nabla T / e B$ as to be the neoclassical prediction in this turbulent regime. The last term is labeled $-\nabla \cdot \Pi_{\mathrm{RS}} / \mu_{i i}=v_{\theta}^{\mathrm{RS}}$ in Fig. 3 and corresponds to the Reynolds stress-driven poloidal velocity, $\mu_{i i} \approx 0.78 \sqrt{\epsilon} \nu_{i i}$ being the ion viscosity and $\nabla \cdot \Pi_{\mathrm{RS}} \propto \rho_{\star}^{2}$. At last, the difference $v_{\theta}^{\mathrm{GYS}}-v_{\theta}^{\mathrm{NC}}$ is labeled $v_{\theta}^{\mathrm{Turb}}$.

The strong correlation between $v_{\theta}^{\text {turb }}$ and $v_{\theta}^{\mathrm{RS}}$ (their discrepancy being a rough measure of how close the system is from a long-time equilibrium) sorely suggests that the observed difference between the complete computation $v_{\theta}^{\mathrm{GYS}}$ and its neoclassical estimate does come from the turbulence. Its contribution can locally range up to 50\% in the actual magnitude of the rotation. These calculations may apply to current tokamaks; when extrapolating to Iter, reliable predictions would require runs at 2 times smaller $\rho_{\star}$ and at least 20 times smaller collisionality $\left(\nu_{\star} \lesssim 2 \times\right.$ $10^{-3}$ ), roughly meaning 20 times longer simulations on a $2^{3}$ larger grid, pushing the envelope on today's most powerful supercomputers. These simulations at smaller ion viscosity may nonetheless be all the more relevant since the turbulent Reynolds stress-induced poloidal velocity $\propto \rho_{\star}^{2} / \nu_{\star} \sim 20 / 2^{2}$ may accordingly increase. This idea is illustrated in Fig. 4 (top), while displaying the averaged poloidal velocities $v_{\theta}^{\mathrm{GYS}}$ and $v_{\theta}^{\mathrm{RS}}$-normalized to the neoclassical value. In the scanned range of collisionality, the magnitude of the poloidal rotation is consistent within $20 \%$ with the neoclassical prediction; however, though the averaged turbulence-induced poloidal rotation is small due to its radial structure, its absolute value is not and increases as $\nu_{\star}$ decreases. It may at last be noted that the cases reported here are that of an homogeneous " $L$-mode"-like plasma with neither ITBs nor strong variations on short distances of the radial electric field or of its derivatives. Accordingly, the foreseen contribution of the Reynolds stresses to $v_{\theta}$ may well here be a lower bound estimate as compared to such more drastic, yet relevant cases.
At last, the corrugation in $v_{\theta}^{\mathrm{NC}}$ (Fig. 3, top) connects to the turbulent modification of the mean temperature profile which in turn sustains a stationary mean $\mathbf{E} \times \mathbf{B}$ flow shear with the same corrugated structure (Fig. 3, bottom). Furthermore, the amplitude of this mean shear is dominant as compared to the usual zonal $\mathbf{E} \times \mathbf{B}$ flow shear (the envelope of its fluctuations over a collision time is pictured in Fig. 3). This result is robustly confirmed throughout the whole scan in $\left(R / L_{T}, \nu_{\star}\right)$ space, as displayed in Fig. 4 (bottom), where the variance of the shear $\sigma(\gamma)=\sum_{i=1}^{n}\left(\gamma_{i}-\right.$ $\left.\langle\gamma\rangle_{r}\right)^{2} /(n-1)\left[\square_{r}\right.$ has $n$ radial points] is plotted for both MFs and ZFs (normalized in each case to the "neoclassical" variance of the shear of $v_{\theta}^{\mathrm{NC}}$ ). These results indicate a prominent role for the mean $\mathbf{E} \times \mathbf{B}$ shear and the turbulence-induced mean profile dynamics; these effects have so far been widely disregarded, considerable focus having been held by fixed equilibrium profiles and zonal fields.

The authors thank $\mathrm{Ch}$. Passeron and acknowledge many stimulating discussions with P.H. Diamond and C.S. Chang and fruitful interactions at the Festival de Théorie in Aix-en-Provence, 2007. This work was performed under the auspices of the European Communities under the contract of Association between EURATOM and CEA. It was carried out within the framework of the EFDA. It also benefited from the support of U.S. Scidac GPS-TTBP center, Grant No. DE-FC02-08ER54959.

[1] A. J. Brizard et al., Rev. Mod. Phys. 79, 421 (2007).

[2] F. L. Hinton et al., Rev. Mod. Phys. 48, 239 (1976).

[3] K. H. Burrell et al., Phys. Plasmas 1, 1536 (1994).

[4] K. Crombé et al., Phys. Rev. Lett. 95, 155003 (2005).

[5] Z. Lin et al., Phys. Rev. Lett. 83, 3645 (1999).

[6] G. Falchetto et al., Phys. Rev. Lett. 92, 025002 (2004).

[7] P. H. Diamond et al., Plasma Phys. Controlled Fusion 47, R35 (2005).

[8] X. Garbet (to be published).

[9] G. Dif-Pradalier et al., Phys. Plasmas 15, 042315 (2008).

[10] V. Grandgirard et al., J. Comput. Phys. 217, 395 (2006).

[11] X. Garbet et al., Phys. Plasmas (to be published).

[12] G. Dif-Pradalier et al., Phys. Plasmas (to be published).

[13] C. S. Chang et al., Phys. Fluids 25, 1493 (1982).

[14] M. Taguchi, Plasma Phys. Controlled Fusion 30, 1897 (1988).

[15] Y. B. Kim et al., Phys. Fluids B 3, 2050 (1991).

[16] F. L. Hinton et al., Plasma Phys. Controlled Fusion 41, A653 (1999).

[17] F. I. Parra et al., Plasma Phys. Controlled Fusion 50, 065014 (2008).

[18] A. M. Dimits et al., Phys. Plasmas 7, 969 (2000).

[19] Y. Sarazin et al. (to be published).

[20] R. Sanchez et al., Phys. Rev. Lett. 101, 205002 (2008).

[21] P. H. Diamond et al., Phys. Plasmas 2, 3640 (1995).

[22] D. E. Newman et al., Phys. Plasmas 3, 1858 (1996).

[23] C. S. Chang et al., Phys. Plasmas 16, 056108 (2009).

[24] M. A. Malkov et al., Phys. Plasmas 8, 3996 (2001).

[25] P. H. Diamond et al., Phys. Rev. Lett. 72, 2565 (1994). 